\title{
THE EFFECT OF AGING UPON RETENTIVE CHARACTERISTICS OF TWO DIFFERENT STUD ATTACHMENTS IN VITRO STUDY
}

\author{
Khaled Aziz Abdelwahab*
}

\begin{abstract}
Aim: In Vitro study was conducted to compare the retention of CM LOC and locator attachment systems in retaining mandibular overdenture upon simulation of insertion-removal cycles.

Materials Abd methods: Ten complete dentures were fabricated on a Stone model representing a completely edentulous mandible that was obtained by making an impression for an edentulous patient. The stone model was duplicated using laboratory addition silicone after placing single implant analogue in the Medline using a dental surveyor. Then the mold was duplicated using epoxy resin. The prefabricated dentures were divided into two groups. Group was set to receive the CM LOC attachment and the other group was set to receive the ball attachment. Using a universal testing machine, the retention was measured at base line, 500, 1000, 1500, and 2000 insertion and removal cycles using ROBOTA chewing simulator, which represents $(0,3,6,9,12)$ month; Considering that the patient will remove the denture 4 to 5 times a day
\end{abstract}

Results: There was a statistically significant difference between the two types of attachments. The CM LOC attachment showed a higher statistically significant mean difference when compared to locator attachment after 1000 cycles (9 months). It was concluded that CM LOC appeared to have a favorable retention over the locator attachment in the first year with less serviceability.

KEYWORDS: Overdentures, overlay dentures, dental attachment, attachment system, implant retained and retention

\section{INTRODUCTION}

Edentulism is defined as complete loss of all natural teeth ${ }^{1}$. As the rate of total edentulism is decreasing in developed countries, the opposite is the case in developing countries; being attributed mainly to the prevalence of periodontal disease and caries. ${ }^{2}$ Conventional complete dentures have been used for years as a treatment option for edentulous patients ${ }^{3-4}$.
Movement of the denture also leads to negative effect on esthetics and phonetics together with social embarrassment. Hence, authors considered the use of implant supported overdentures can help to overcome the problems of conventional complete denture $^{5-9}$.

The high costs and the invasive nature of implant surgeries are considered major limiting factors

\footnotetext{
* Associate Professor, Department of Prosthodontics, Cairo University, New Giza University.
} 
for wide spread acceptance of implant supported overdentures. Single implant-retained overdentures have gained popularity in the past years due to their lowered costs and minimal tissue trauma. In 2017 Nicol Passia and his colleagues conducted a multicenter randomized controlled trial to investigate the masticatory performance of patients with overdentures retained by one implant in the midline and found a significant in the increases masticatory performance ${ }^{10}$

There are different attachments with different types of materials of matrices that can be used nowadays such as; Nylon in locators, Teflon in ball and socket, and poly-etherketoneketone that were recently used for the CM LOC attachment system. Poly-etherketoneketone is a family member of poly-aryletherketones (PAEKs). PAEKs are a group of high-performance semi-crystalline thermoplastic resins that have been successfully applied in different medical applications such as frameworks for fixed and removable prosthesis as well as attachment matrices.

Thus, the question here, which type of attachment have long term retention as well as less maintenance properties?

\section{MATERIALS AND METHODS}

A Stone model representing a completely edentulous mandible was obtained by making an impression for an edentulous patient. The shape of the mandible was selected to allow the placment of the implant straight in the midline.

The stone model was duplicated into epoxy resin model. The obtained duplicate was poured into stone model ten times to fabricate ten identical complete dentures. Acrylic resin teeth with a proper size were set up on a trial denture bases, which was waxed up on the constructed stone models. A putty index was constructed to cover all the teeth and polished surface. This index will be used after wards to duplicate the artificial teeth setup as well as the waxing up in all dentures used in this study.

The epoxy model was drilled in the midline area using a milling surveyor, to create an oversized hole. The implant mount was attached to the milling surveyor to ensure orthogonal placement of the implant analogue* $\left(3.5^{*} 10 \mathrm{~mm}\right)$ in the oversized hole. The implant analogue was placed in the prepared hole until its crestal module was flushed with the surface of the stone model. Afterwards, the implant was glued in place using self-cured acrylic resin (Fig 1A). The waxed-up dentures were then processed and polished. The finished dentures were placed on the epoxy model to ensure fitness

The ten prefabricated dentures were divided into two groups $\mathrm{A}$ and $\mathrm{B}$, each group contains five dentures. Group A was set to receive the $\mathrm{CM} \mathrm{LOC}^{* *}$ attachment and group B was set to receive the ball attachment $^{* * *}$. CM LOC attachment consists of a titanium patrix that was attached to the implant analogue and a matrix made from polyetherketoneketone (PEKK) in a metal cap housing. While the ball and socket attachment made of a titanium abutment was screwed to the implant analogue together with a nylon cap in a metal housing both housings were picked up in the fitting surface of the corresponding denture using Self-curing acrylic ${ }^{* * * *}$.

Geometric center was marked at which dislodging forces will be applied by connecting the anterior and posterior extremes of the denture (midline and retromolar pads respectively) to form a triangle, the intersection of the three lines bisecting the three angles of the triangle was considered as the geometric center. On the other hand, a tripod steel rod was prepared and attached to the denture using auto polymerizing acrylic resin (Fig. 1B)

\footnotetext{
* Zimmer, Biomet USA

** CM LOC® cendres \&metaux, Swizraland

*** Zimmer, Biomet USA

**** Acrostone Dental \& Medical Supplies, Heliopolis, Cairo, Egypt
} 
ROBOTA Chewing simulator ${ }^{*}$ (Fig. 3) is a newly developed machine, it has four chambers with the ability to perform chewing cycles as well as insertion and removal cycles. These cycles can be performed in wet environment simulating the saliva (Isotonic $0.9 \%$ sodium chloride solution at $22^{\circ} \mathrm{C}$ ). Mechanical aging of materials can be performed through cyclic loading through a programmable logic-controlled equipment with thermo-cyclic protocol operated on servo-motor. Each chamber consists of an upper Jackob's chuck as a steel stylus holder that was be tightened with a screw and the Teflon housing lower part as a sample holder.

The epoxy model was attached to the lower Teflon housing by means of a tightening screw. On the other hand, the overdenture's tripod steel was attached to the upper Jackob's chuck as a steel stylus holder that can be tightened with a screw. A weight of $5 \mathrm{~kg}$, which is comparable to $49 \mathrm{~N}$ of chewing force was exerted, with a vertical up and down movements of the upper compartment. These vertical movements should be able to detach the metal housing with the retentive part away from the patrix. The test was repeated 2000 times of insertion/removal cycles according to previously performed studies ${ }^{11}$ (Fig 2). This test was performed to every denture in each group (A and B).

Retention tests were performed using universal testing machine Bluehill Lite; Instron Instruments** with a load cell of $5 \mathrm{KN}$ Retention was assessed at the baseline, after 500 cycle, 1000 cycle, 1500 cycle and 2000 cycle). Data were recorded using computer software ${ }^{* * * *}$.

The upper plate of the machine included specially designed retention measuring device that grasped a steel stylus of each denture, while a hole made in the epoxy model to be fixed to the lower compartment by tightening screw, to prevent its dislodgment during the application of tensile force. Tensile forces were applied to the overdentures through the tripod steel, which was attached to the universal testing machine. The device was subjected to a slowly increasing vertical load $(50 \mathrm{~mm} / \mathrm{min})$ until total dislodgment of the denture, accompanied by decline in load-displacement curve recorded by Bluehill Lite software. This was done for each attachment separately. Data were recorded by Bluehill Lite computer software in Newton force and maximum load was recorded. The obtained data was tabulated and subjected to statistical analysis

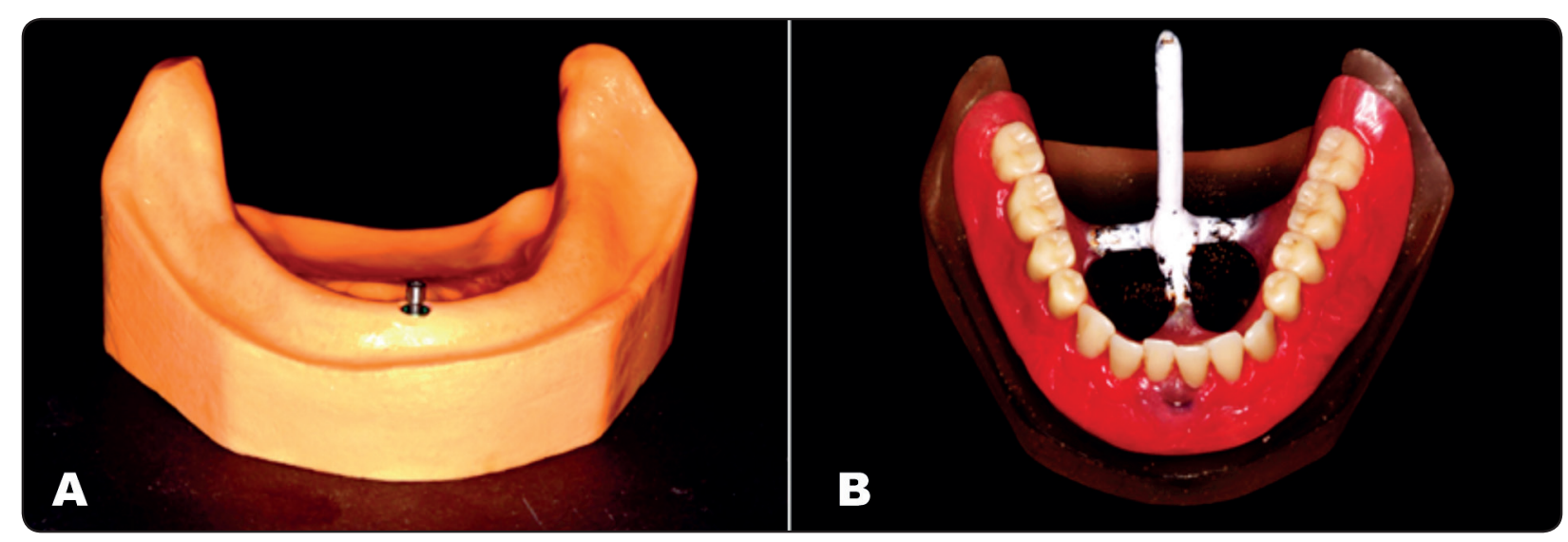

Fig. (1) A) Implant analogue in place in the model. B) Denture geometric center.

\footnotetext{
* Model ACH-09075DC-T, AD-Tech Technology CO., LTD., Germany

** Model 3345; Instron Industrial Products, Norwood, USA

*** Bluehill Lite; Instron Instruments
} 


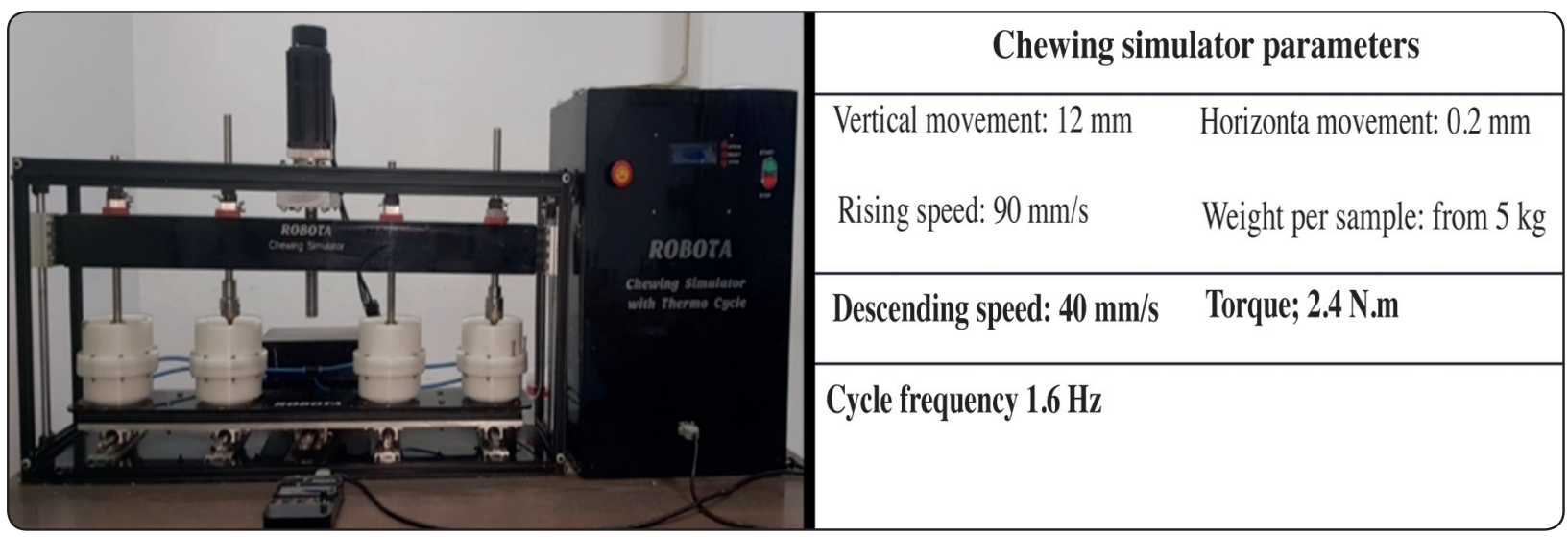

Fig. (2) ROBOTA chewing simulator

\section{RESULTS}

Data were analyzed using IBM SPSS advanced statistics $^{\circledast}$ (Statistical Package for Social Sciences). Numerical data will be described as means and standard deviation. The data were explored for normality using Kolmogorov-Smirov test. Comparisons between the two studied groups were done by independent sample t-test. Repeated measures analysis of variance (ANOVA) test was performed to compare the studied variable within each group. Post Bonferroni hoc test was used to detect the significant variable. In all the previous tests the results were considered statistically significant if a p-value $<0.05$.

\section{I) CM LOC along different time intervals}

When comparing the base line reading with the $500,1000,1500$ and 2000 cycles, it was found that there is no statistical difference at 500 and 1000 cycles. Statistical differences were found at 1500 and 2000 cycles with $\mathrm{P}$ value $<0.01$.

\section{II) Ball ad socket along different time intervals}

When comparing the base line reading with the $500,1000,1500$ and 2000 cycles, it was found that there is statistical difference at 500, 1000, 1500 and 2000 cycles with $\mathrm{P}$ value $<0.01$.

\section{III) CM LOC vs ball and socket}

Statistical analysis of the long term retentive characteristics of the CM LOC and ball attachment showed a significant difference between them after 1000 cycles (9 months) ${ }^{12}$ CM LOC attachment showed a higher statistically significant mean difference when compared to ball attachment.

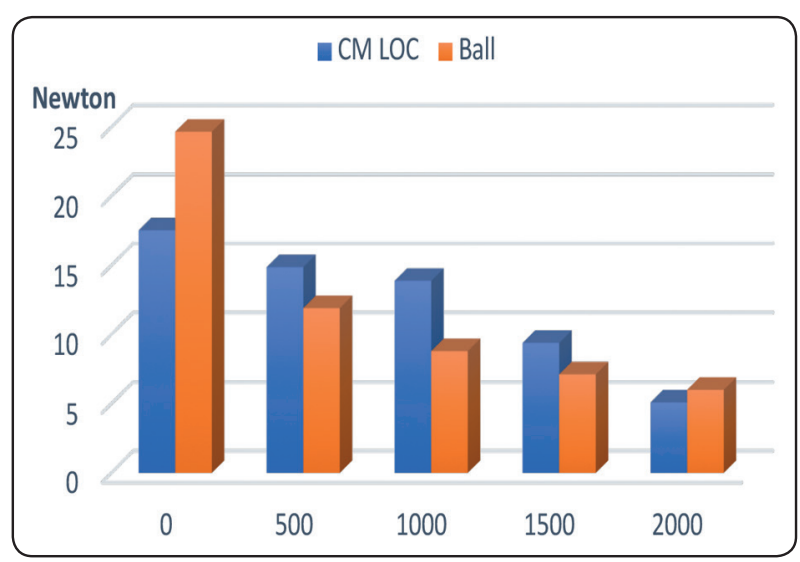

Fig. (3) Graph representing retention values of CM LOC vs ball and socket attachment: 


\section{DISCUSSION}

Edentulous patients suffer a lot from their conventional complete dentures. Their problems are most commonly associated with the mandibular dentures due to lack of stability and retention. By the introduction of implants, implant-supported overdentureisconsideredausefultreatmentoptionfor compromised mandibular ridge ${ }^{13}$.Many anchorage systems had been suggested for retaining implantsupported overdentures including ball and socket, bars and magnetic attachments ${ }^{7,8}$. The differences in the retentive capacities between different types of dental attachments is still regarded a controversial issue in the prosthodontic literature. Up to date, the minimal amount of retention necessary to obtain adequate patient satisfaction is not yet concluded ${ }^{14}$. Ball and socket attachments are the most commonly used type of attachment because of its low cost and less complicated laboratory procedures ${ }^{15}$.

In this study, two types of attachments; CM LOC and ball attachment (Zimmer) were tested for long term retention using a universal testing machine after being subjected to mechanical aging through insertion/removal cyclic loading via ROBOTA chewing simulator.

The results of this study revealed that the ball group (B) has yielded higher initial retention when compared to the CM LOC group (A). Both attachments reduced its retention by time especially after 2000 cycles which refers to 1.5 year of usage. When comparing the base line reading of the $\mathrm{CM}$ LOC with the 500, 1000, 1500 and 2000 cycles, it was found that there is no statistical difference at 500 and 1000 cycles. Statistical difference was found at 1500 and 2000 cycles. While the ball attachment shows a significant difference between the base line and the 500, 1000, 1500 and 2000 cycles. Although most types of overdenture attachments usually require maintenance after the first year of use, fortunately; the CM LOC attachment system was found to yield adequate retention after the 9 months of use with only $22 \%$ loss of retention. On the other hand, the ball attachment group resulted in $65 \%$ retention loss after 1000 cycles, which approximately corresponds to 9 months of use.

These results might be attributed to the nature of the CM lock matrix which have a slot in matrix of the CM LOC attachment system, which expands when the matrix and the patrix come in contact. The expansion that occurs reduces the deterioration of the surface of the matrix, thus minimizing the resultant wear $^{17}$.

\section{CONCLUSION}

Within the limitation of invitro study, we can conclude that both attachments showed a satisfactory retentive force at the base line as well significant decrease in retention throughout one and a half year of time. CM LOC appeared to have a favorable retention over the ball attachment in the first year with less serviceability. Within the limitations of this investigation, the combination of a titanium patrix and a matrix made from polyetherketoneketone with a slot preparation seems to be favorable for long-term retention. PEKK material could be used as a matrix material for attachment

\section{REHRERANCE}

1. Driscoll CF, Freilich MA, Guckes AD, et al. The Glossary of Prosthodontic Terms.

2. Nalçaci R, Erdemir EO, Baran I. Evaluation of the oral health status of the people aged 65 years and over living in near rural district of Middle Anatolia, Turkey. Arch Gerontol Geriatr.

3. Critchlow SB, Ellis JS. Prognostic indicators for conventional complete denture therapy: A review of the literature.

4. Tallgren A. The continuing reduction of the residual alveolar ridges in complete denture wearers: A mixed-longitudinal study covering 25 years.

5. Atwood DA. Reduction of residual ridges: A major oral disease entity.

6. DOUNDOULAKIS JH, ECKERT SE, LINDQUIST CC, JEFFCOAT MK. The implant-supported overdenture as an alternative to the complete mandibular denture. 
7. Assaf A, Daas M, Boittin A, Eid N, Postaire M. Prosthetic maintenance of different mandibular implant overdentures: A systematic review.

8. D. Mericske-Stern R, Taylor TD, Belser U. Management of the edentulous patient.

9. Fueki K, Kimoto K, Ogawa T, Garrett NR. Effect of implant-supported or retained dentures on masticatory performance: A systematic review.

10. Passia N, Abou-Ayash S, Reissmann DR, et al. Single mandibular implant study (SMIS) - masticatory performance - results from a randomized clinical trial using two different loading protocols.

11. Choi JW, Bae JH, Jeong CM, Huh JB. Retention and wear behaviors of two implant overdenture stud-type attachments at different implant angulations.

12. Mattia PR, Botega DM, Frasca LCF. Evaluation of reten- tive force of attachment systems for overdentures retained by one or two implants.

13. Yan Q, Hua F, Shi B. Patients Are More Satisfied With Implant-Supported Mandibular Overdentures Than With Conventional Dentures.

14. Burns DR, Unger JW, Coffey JP, Waldrop TC, Elswick RK. Randomized, prospective, clinical evaluation of prosthodontic modalities for mandibular implant overdenture treatment.

15. Manju V, Sreelal T. Mandibular Implant-Supported Overdenture: An In Vitro Comparison of Ball, Bar, and Magnetic Attachments.

16. Kobayashi M, Srinivasan M. Effects of in vitro cyclic dislodging on retentive force and removal torque of three overdenture attachment systems.

17. Passia N, Ghazal M, Kern M. Long-term retention behaviour of resin matrix attachment systems for overdentures. 